\title{
Rede de Atenção à Saúde: integração sistêmica sob a perspectiva da macrogestão
}

\author{
Healthcare network: systemic integration from the perspective of \\ macromanagement
}

Edivânia Lucia Araujo Santos Landim', Maria do Carmo Lessa Guimarães², Ana Paula

Chancharulo de Morais Pereira ${ }^{3}$

DOI: 10.1590/0103-11042019S514

RESUMO Este artigo analisa a integração sistêmica da atenção à saúde da linha de cuidado do câncer de mama, no contexto da regionalização da saúde no estado da Bahia, sob a perspectiva da macrogestão. Estudo transversal retrospectivo, de natureza exploratória e descritiva, com uso da abordagem qualiquantitativa e referencial da rede de políticas públicas. Foram utilizadas as técnicas de análise documental, extração de dados dos sistemas de informação, entrevistas semiestruturadas com 141 participantes na coleta de dados. Elegeram-se como categorias analíticas: Desenho institucional da rede de oncologia; Atenção Primária à Saúde como porta de entrada e ordenadora da rede; Sistemas de apoio; e Sistemas logísticos. Os resultados sinalizaram que as normas, embora necessárias, não são per si suficientes para garantir a integração sistêmica; o desenho de redes de atenção à saúde tem seguido a lógica de estruturação por oferta, adensando-se nos grandes centros populacionais, gerando vazios assistenciais; predomina o acesso da população aos serviços não complementares ao Sistema único de Saúde (SUS) e/ou de alta densidade tecnológica, evidenciando a hegemonia do modelo médico-centrado e privatista; mecanismos de regulação do acesso e de gestão dos sistemas de informação ainda ocorrem de forma incipiente, com pouca e/ou esparsa interação entre si.

PALAVRAS-CHAVE Integração. Redes. Atenção à saúde.

1 Secretaria da Saúde do Estado da Bahia (Sesab) Salvador (BA), Brasil. vanialandim@hotmail.com

2 Universidade Federal da Bahia (UFBA) - Salvador (BA), Brasil.

ABSTRACT This article analyzes the systemic integration of health care in the breast cancer care scope, in the context of the regionalization of health in the state of Bahia, under the perspective of macromanagement. Retrospective cross-sectional study, exploratory and descriptive, using the quali-quantitative and referential approach of the public policy network. The techniques of documentary analysis, data extraction from information systems, and semi-structured interviews carried out with 141 participants in the data collection were used. The chose analytical categories were: Institutional design of the oncology network; Primary Health Care as gateway to the network; Support systems; and Logistics systems. The results showed that the standards, although necessary, are not sufficient in themselves to guarantee systemic integration; the design of health care networks has followed the logic of structuring by supply, becoming denser in large population centers, generating care vacancies; the population's access to services that are not complementary to the Unified Health System (SUS) and/or high technological density prevails, evidencing the hegemony of the medicalcentered and privatized model; mechanisms of regulation to access and management of information systems still occur in an incipient way, with little and/or sparse interaction between them.

KEYWORDS Integration. Networks. Health care.

3 Universidade do Estado 


\section{Introdução}

O desenho inicial do Sistema Único de Saúde (SUS), ao privilegiar a estratégia de descentralização sem integração regional, pulverizou e fragmentou a oferta de serviços, gerando vazios assistenciais no território brasileiro, ao mesmo tempo que fragilizou a capacidade dos governos estaduais, no tocante aos seus papeis de coordenador e regulador da rede de saúde $e^{\mathbf{1 , 2}}$.

Com o propósito de aperfeiçoar a dinâmica político-institucional do SUS e de superar a fragmentação da atenção, o Ministério da Saúde (MS) publicou a Portaria ${ }^{0} 4.279 / 2010^{3}$, que estabeleceu as diretrizes para a organização da Rede de Atenção à Saúde (RAS), tendo como objetivo a integração sistêmica das ações e serviços de saúde ${ }^{\mathbf{4}, 5}$.

Contudo, as linhas de integração dos serviços obedecem a lógicas territoriais que extrapolam as suas fronteiras. O processo encontra-se sujeito às ingerências do capital econômico e do poder político-partidário, acentuando as assimetrias nas relações de poder, com concentração de recursos e tecnologias em algumas regiões de saúde. Acrescenta-se a forte presença do mix público-privado na oferta de serviços, a qual pode atuar como facilitador ou entrave na organização de redes integradas e regionalizadas ${ }^{6,7}$.

Embora o Decreto Presidencial $n^{0} 7.508 / 11^{8}$ reafirme a importância da regionalização no seu arcabouço normativo, incluindo o planejamento e a assistência à saúde, a articulação interfederativa, a estruturação das RAS e a trajetória de institucionalidade do SUS têm mostrado que os instrumentos jurídico-normativos, ainda que sejam importantes, não são, per si, suficientes para superar as iniquidades do sistema de saúde e as desigualdades intra e inter-regionais do território brasileiro ${ }^{9}$. A reprodução da lógica de modelos homogêneos e inflexíveis, sem admitir a dimensão política desse processo, tende a superestimar o poder normativo para alterar cenários sociais e políticos institucionais.

Esses elementos de contexto geram constrangimentos à implementação e à gestão das
RAS, conforme prevista pela normativa do SUS, defendida como alternativa conceitual e operacional, para a oferta de ações e serviços de distintas densidades tecnológicas, capazes de garantir a integralidade do cuidado, um dos princípios basilares do SUS. Além disso, tal situação imprime diferentes institucionalidades à estratégia de gestão regional das redes integradas de atenção à saúde, em âmbito nacional, o que torna o processo de integração sistêmica das ações e serviços de saúde um fenômeno político, complexo, plural e, ao mesmo tempo, singular.

Por sua vez, a materialidade da imagem-objetivo de um sistema de redes integradas de saúde tem-se mostrado difícil em sua implementação, demandando, portanto, estudos que contribuam para a sua efetiva implantação ${ }^{10}$. Nessa direção, pesquisa de revisão do estado da arte constatou a escassez de estudos empíricos nesse campo e afirmou o grande desafio para gestores e pesquisadores analisarem os efeitos da integração dos serviços de saúde ${ }^{11}$.

Entende-se que a integração sistêmica remete invariavelmente ao princípio constitucional de integralidade da atenção à saúde, equidade e universalidade do acesso. Constitui-se ainda em uma prática social e em uma luta política pela reafirmação do projeto do SUS ${ }^{10,12}$, tensionando e imprimindo uma dinâmica entre o campo do instituído e do instituinte, do normativo e do experienciado cotidianamente nas organizações de saúde, nos seus modos de produzir saúde.

Diante do exposto, admite-se que a integração sistêmica das ações e dos serviços de saúde, no âmbito das RAS, consiste em redes interorganizacionais e intraorganizacionais, coordenadas e articuladas, para organizar serviços de diferentes densidades tecnológicas em uma região adscrita e gerir os distintos pontos de atenção à saúde que integram a linha de cuidado. A centralidade é o usuário, de forma a promover a acessibilidade geográfica e sócio-organizacional e garantir a integralidade do cuidado, observando as noções de espaço e tempo para intervenção oportuna ${ }^{13}$. 
Embora a abordagem da gestão de redes de política induza à noção de coordenação e mediação de relações intra e interorganizacionais para formulação e implementação de políticas públicas, isso não significa afirmar que o campo organizacional seja destituído de uma intencionalidade e de relações de poder e posições entre seus distintos atores. Por outro lado, a integração das ações e dos serviços não pode ser vista como uma variável dicotômica da fragmentação ${ }^{10}$, mas como diferentes graus ou formas de institucionalidade, considerando-se a dimensão política, a dinamicidade e a mutabilidade do processo.

Os seguintes pressupostos nortearam este estudo: o desenho institucional de redes de atenção à saúde estrutura-se sob a lógica da oferta, gerando vazios assistenciais cujos mecanismos de regulação de acesso ainda ocorrem de forma incipiente, com pouca e/ ou esparsa interação entre si, culminando por impulsionar o acesso da população à rede não complementar ao SUS; problemas gerenciais de concepção, interoperabilidade e defasagem dos dados comprometem a gestão integrada dos sistemas de informações.

Destarte, o objetivo deste estudo é analisar a integração sistêmica da atenção à saúde da linha de cuidado do câncer de mama, no contexto da regionalização da saúde na Bahia, sob a perspectiva da macropolítica.

A escolha dessa linha de cuidado decorre do fato de ser tratada como uma ação estratégica e prioritária do governo da Bahia, aliada ao processo de transição sociodemográfica e epidemiológica que vem ocorrendo no Brasil e no mundo, que resulta no envelhecimento da população e no aumento das condições crônico-degenerativas.
No Brasil, em 2018, estimou-se a ocorrência de 600 mil novos casos de câncer, sendo os mais frequentes os de próstata e de mama, respectivamente, com 68.220 casos em homens e 59.700 em mulheres ${ }^{14}$. A Bahia segue essa tendência, sendo o câncer de mama o de maior incidência em mulheres, constituindo-se na primeira causa de mortalidade no segmento feminino ${ }^{15}$.

\section{Material e métodos}

Trata-se de um estudo transversal retrospectivo, de natureza exploratória e descritiva, com uso da abordagem quantitativa e qualitativa. Adotou-se o referencial teórico da rede de políticas públicas. O modelo teórico-lógico adotado ancora-se também nos fundamentos da integração sistêmica para a integralidade do cuidado (integração normativa, funcional, clínica e do cuidado) de Hartz e Contandriopoulos ${ }^{10}$ e de acessibilidade aos serviços de saúde, adaptando-se o modelo de Cunha e Vieira-da-Silva ${ }^{16}$, no tocante ao acesso geográfico e sócio-organizacional.

Tendo em vista o objetivo da pesquisa, fez-se um recorte dos elementos constitutivos da RAS, com foco para alguns elementos da estrutura operacional (figura 1), cuja matriz do modelo teórico-lógico elencou, para efeitos analíticos da gestão de redes integradas e regionalizadas, duas dimensões que se inter-relacionam e se interpenetram: a macro e a micropolítica. Essas dimensões são compostas, respectivamente, da 'organização das RAS nas regiões de saúde' e da 'gestão da rede de serviços de saúde'. Neste artigo, a dimensão da macrogestão foi tomada como objeto de análise. 
Figura 1. Estrutura do texto normativo da Portaria GM/MS no 4.279/2010

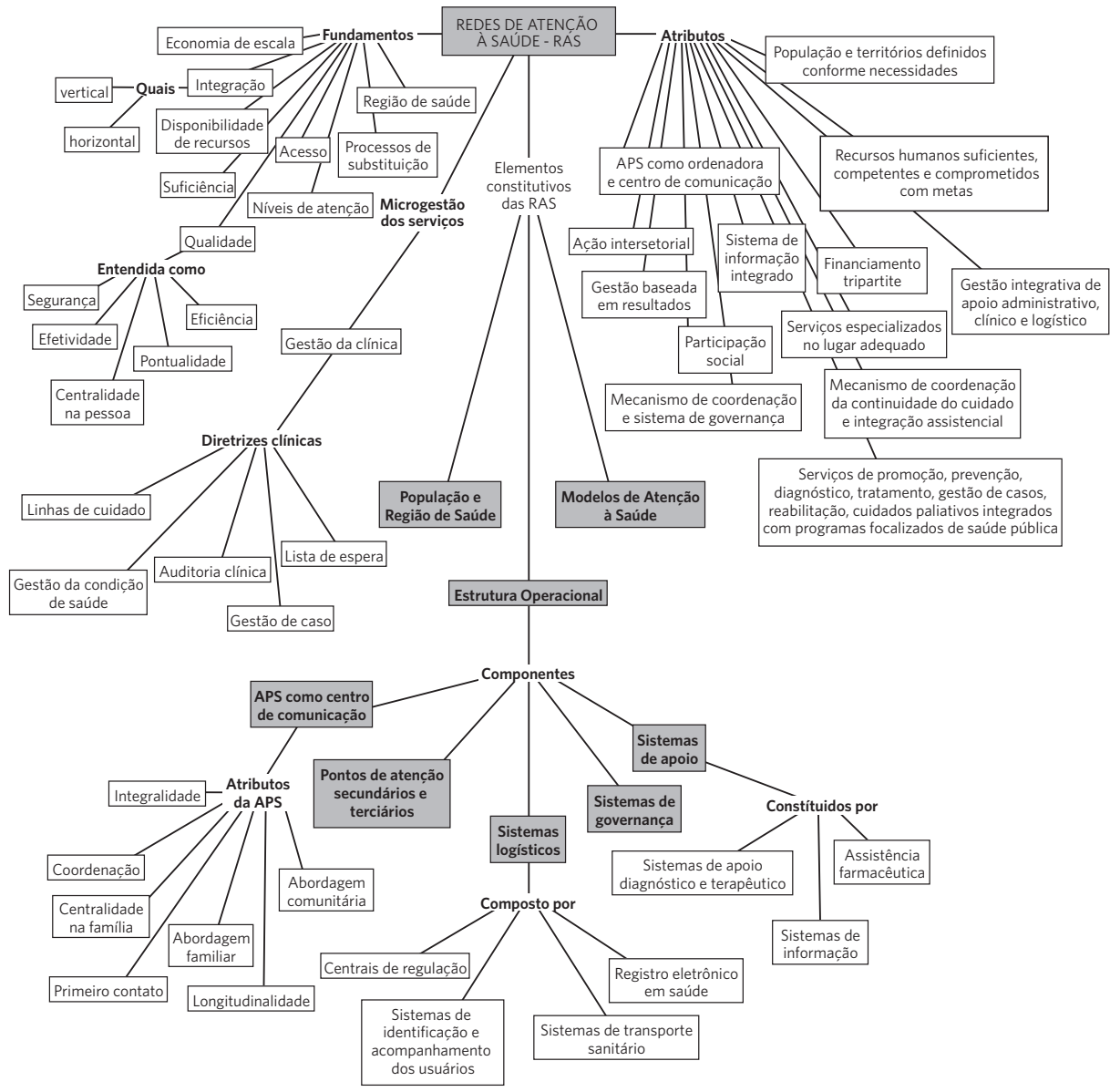

Fonte: Landim"1.

A subdimensão 'organização das RAS nas regiões de saúde' envolve os seguintes componentes: desenho institucional da rede de oncologia no estado da Bahia (diretrizes políticas, distribuição dos serviços, abrangência regional e cobertura populacional); Atenção Primária à Saúde (APS) como porta de entrada, coordenadora do cuidado e ordenadora da rede; sistemas de apoio, relacionados com os sistemas de informação em saúde, como o Registro Hospitalar de Câncer (RHC), Registro de Câncer de Base Populacional (RCBP) e Sistema de Informação do Câncer (Siscan) e sua interoperabilidade, existência de sistema de informação que interligue todos os pontos de atenção da rede interorganizacional da linha de cuidado; sistemas logísticos (suporte estrutural de casas de apoio ou similares; mecanismos de regulação do fluxo de acesso aos serviços, mediante transporte sanitário das(os) usuárias(os) e acompanhantes, sistemas de marcação de consulta e/ou exames de diagnóstico).

Foram utilizados como fontes e instrumentos de coleta de dados: a técnica de análise documental (planos, relatórios técnicos, arcabouço jurídico-normativo do SUS); dados extraídos do RHC, do RCBP e do Siscan; e entrevistas semiestruturadas, com uso da história da narrativa oral para compreensão 
dos núcleos de sentido. O instrumento da entrevista semiestruturada foi previamente testado, mediante amostragem acidental ou por conveniência, com cerca de 35 pacientes do sexo feminino.

Após a etapa de validação, a pesquisa foi realizada em março de 2018, com 139 (98,5\%) mulheres e $2(1,41 \%)$ homens, totalizando 141 participantes, oriundos de 76 municípios, incluindo a capital do estado, correspondendo a 18,2\% do total dos 417 municípios baianos. Da totalidade de participantes, 101 são procedentes do interior do estado; e 40, do município de Salvador, representando, este último, 28,3\% da amostra. Dos 101 participantes do interior da Bahia, verifica-se que são oriundos de 8 , do total de 9 macrorregionais de saúde, conforme Plano Diretor de Regionalização (PDR). Nessa fase da pesquisa, fez-se uso da amostragem intencional para atrair participantes não residentes no município de Salvador, muito embora a presença de usuárias(os) procedentes da capital fosse significativa, em decorrência da acessibilidade geográfica, entre outros fatores.

Referente à escolaridade das(os) participantes, $48,2 \%$ possuíam ensino médio incompleto; $28,3 \%$, ensino médio completo, cujo somatório do número de semialfabetizadas(os) e analfabetas(os) correspondeu a 19,1\%. Participantes com graduação incompleta, completa e pós-graduação perfizeram $4,25 \%$.

As entrevistas foram realizadas no ambulatório de mastologia do único Centro de Assistência de Alta Complexidade em Oncologia (Cacon), referência para todo o território baiano para cânceres prevalentes e raros, cuja unidade hospitalar aporta o maior número de usuárias(os) da capital e interior do estado, conforme dados do RHC, fato que motivou a escolha dessa unidade de saúde. Os entrevistados leram e assinaram o Termo de Consentimento Livre e Esclarecido (TCLE) em duas vias.

Em observância à Resolução n ${ }^{0} 466 / 2012^{17}$, do Conselho Nacional de Saúde, o estudo foi submetido à apreciação de dois Comitês de Ética em Pesquisa, obtendo-se parecer favorável, respectivamente, $\mathrm{n}^{\circ} 2.349 .858$, em 27 de setembro de 2017 e n ${ }^{\circ} 2.444 .577$, em 18 de dezembro de 2017.

\section{Resultados e discussão}

São apresentados os resultados da dimensão macropolítica e respectivos subcomponentes em subseções, ressaltando-se a interconexão entre eles, de acordo com as categorias identificadas nos dados coletados: Desenho institucional da rede de oncologia; APS como porta de entrada e ordenadora da rede; sistemas de apoio e logísticos.

\section{Desenho institucional da rede de oncologia}

O Plano Estadual de Atenção ao Câncer 2016$2023^{18}$, aprovado na Comissão Intergestores Bipartite (CIB), conforme Resolução $n^{\circ}$ 170/2015, estabelece, entre seus princípios e diretrizes, a organização de redes de atenção regionalizadas e descentralizadas, com pontos de atenção integrados, em observância aos critérios de acesso, escala e escopo para oferta do cuidado integral em tempo oportuno.

A Rede de Atenção ao Câncer no Estado da Bahia encontra-se presente em 6 das 9 macrorregiões de saúde, totalizando 13 serviços, sendo 6 (46,1\%) localizados na cidade de Salvador; e 7, em municípios-sede de regiões de saúde, para atender uma população estimada em 15.344.447 habitantes ${ }^{19}$, distribuída nos 417 municípios que compõem sua base territorial.

Essa rede organiza-se ainda sob a perspectiva da oferta de serviços existentes no território, com concentração dos serviços na Macrorregião Leste e de vazios assistenciais na Macrorregiões de Saúde Oeste, Centro-Norte e Nordeste ${ }^{18}$, o que evidencia os desafios de acessibilidade geográfica, considerando-se a extensão territorial da Bahia de $564.7733 \mathrm{~km}^{2}$. Cerca de $20 \%$ dos participantes da pesquisa percorrem distâncias entre $300 \mathrm{~km}$ e $400 \mathrm{~km}$; e $33,3 \%$, de $400 \mathrm{~km}$ a $1.000 \mathrm{~km}$, para realizar do tratamento na capital do estado. 
O Plano tem vigência até 2023, e seu escopo contempla desenho de expansão da rede, por meio da implantação/implementação de Unidades de Alta Complexidade em Oncologia (Unacon) e Cacon, mediante a contratualização de novos serviços de saúde. As unidades móveis da Estratégia Itinerante de Saúde integram a proposta de atenção ao câncer. Essa estratégia tem como propósito suprir os vazios assistenciais. Sua rede é $100 \%$ contratualizada, com responsabilidade para circular nas regiões de saúde e assistir parcela da população na faixa etária de 50 anos a 60 anos.

Embora a Estratégia Itinerante de Saúde seja uma ação complementar à Rede Estadual de Atenção ao Câncer de Mama, seus ciclos duram mais de cinco anos sem que se completem. Entretanto, ao priorizar determinado público, em observância à normativa, tende a desassistir parcela significativa da população que se encontra também em situação de vulnerabilidade social, econômica e estrutural. Ademais, o atraso na devolução dos resultados de diagnóstico torna-se entrave para a realização do tratamento em tempo oportuno, conforme se observa nas narrativas.

Esperei por quase um ano para receber a mamografia. É tempo, muito tempo, e a gente fica sem poder fazer nada. (Participante 122).

Eu passei foi quatro meses para receber o resultado da mamografia. Como pode passar quatro meses para receber um exame tão importante como esse? (Participante 124).

Só fui receber o resultado do exame depois de seis meses. Quem já viu isso?! (Informante 140).

No município não se consegue fazer mamografia pelo SUS antes dos 50 anos, e tive que fazer particular para agilizar e sai até mais barato do que pagar passagem para fazer pelo SUS em outro lugar [...]. (Participante 13).

Iniciei meu atendimento numa clínica particular para agilizar, pois não fazia pelo SUS antes dos
50 anos. Depois a Secretaria de saúde fez o encaminhamento para cá [...]. (Participante 52).

Os achados documentais apontam para um formato de rede contratualizada, confirmando a suposição de desconcentração mediante a compra de serviços dos prestadores, sem que isso signifique eficiência, eficácia e efetividade na redução das iniquidades socioespaciais, haja vista a permanência das lacunas assistenciais.

Do ponto de vista da relação entre planejamento regional e desenvolvimento, percebe-se que o modelo de redes vigente aponta que os serviços de saúde, enquanto capital econômico e social, privilegia regiões de grande adensamento populacional supostamente por questões de economia de escala e escopo. Isso implica que municípios de grande porte tendem a concentrar serviços de alta densidade tecnológica, possivelmente orientados por uma lógica de custo-benefício com base em produtividade. Isto, entretanto, pode dissociar-se do princípio que rege o direito à saúde, quando são mantidos os vazios assistenciais e/ou imposições de acesso geográfico. A coexistência desses fatores afeta a universalização do acesso à atenção à saúde com equidade e integralidade.

Esse cenário reabre a discussão acerca do processo de regionalização. Em tese, seria um vetor de planejamento regional e desenvolvimento para superação das desigualdades intra e inter-regionais $\mathbf{1}^{\mathbf{1 6}, \mathbf{2 0}}$, rompendo com o ciclo vicioso de iniquidades e assegurando a universalidade do acesso com equidade e integralidade do cuidado, além de promover qualidade de vida e saúde da população. Sob essa perspectiva, a organização de redes, no território regional, ultrapassaria os limites da distribuição espacial de serviços, uma vez que se vincularia às políticas públicas de desenvolvimento regional, para reduzir essas assimetrias históricas e estruturais.

A configuração da Rede de Atenção ao Câncer, conforme documentos analisados, está condicionada aos serviços existentes no território e ao processo de negociação e pactuação regional, por meio das Comissões 
Intergestoras Regionais (CIR), para implantação das novas unidades assistenciais, incluindo o interesse e a disponibilidade dos prestadores para ofertar esse tipo de atendimento. Essa situação confirma o caráter político do processo de implementação de redes de saúde, ao mesmo tempo que sinaliza uma tendência de capitalização do setor ${ }^{21}$, com predisposição para manutenção e/ ou ampliação da relação público-privada, para provisão dos serviços de saúde à população. $\mathrm{O}$ privilegiamento desse formato revela uma forte inclinação para manter o mix público-privado ${ }^{6,7}$ em uma área da saúde pública em que os procedimentos são de alto custo. Isso, entretanto, a torna extremamente atrativa para o setor privado não complementar ao SUS, retroalimentando o ciclo de financeirização do setor saúde, dependência das ações estatais do capital privado e estabilidade sanitária aos agentes do mercado.

Por sua vez, a gestão de 'redes de redes' de políticas, mediante a diversidade de atores, organizações e diferentes arranjos de gestão, justificada, em termos teóricos, para a eficácia na implementação de políticas públicas, em face dos problemas complexos, não significa que esse movimento seja destituído de uma intencionalidade. Além disso, configura-se exatamente nos moldes idealizados de padrões de interação, comunicação, compartilhamento e interdependência entre os distintos atores. Por outro lado, não se pode afirmar que a estrutura de redes não possua, em seu construto lógico e político-ideológico, valores da reforma administrativa de eficiência, eficácia, efetividade e ganhos de escala. Ao analisar a normativa das RAS e a Resolução do Conselho Intergestores Tripartite $\mathrm{n}^{\circ} 34$, de março de 2018, constatou-se que esses princípios estão presentes e bem articulados.

Até porque a estruturação das RAS fundamenta-se em princípios da teoria econômica de escala e escopo, cujo primado do custo-efetividade oriundo do sistema de mercado norte-americano colide com a tríade indissolúvel de universalidade, integralidade e igualdade ${ }^{22}$.

Observa-se que o desenho institucional da rede de oncologia, no contexto da regionalização da saúde na Bahia, ainda é um processo em construção, assim como acontece em outras unidades da Federação brasileira ${ }^{22}$. Desse modo, tal como o próprio modelo, reproduz a lógica dominante de atenção médico-centrada, tecnicista e privatista, haja vista o boicote passivo ao SUS, mediante o subfinanciamento da rede própria e a valorização do setor privado com subsídios, desonerações e financiamento público ${ }^{23}$.

\section{Atenção primária à saúde como por- ta de entrada e ordenadora da rede}

Os dados revelaram que a APS não tem assumido o papel de 'porta de entrada', coordenadora e ordenadora da rede, no sentido de longitudinalidade e direcionalidade do cuidado, conforme prevê a normativa das RAS. Do total de 141 participantes, apenas 38 (26,9\%) declararam ter iniciado o percurso terapêutico na APS.

A Bahia apresenta uma cobertura das Equipes de Saúde da Família (EqSF) de 73,4\%, abaixo do percentual da região Nordeste, com $80,94 \%$. Do total de municípios de domicílio dos participantes da pesquisa, 62 (81,5\%) possuem cobertura de $74,12 \%$ a $100 \%{ }^{24}$. Contudo, essa relação não se mostrou correspondente em termos de elegibilidade da APS como porta de entrada.

Apesar das funções e atributos da APS, cuja resolutividade situa-se entre $80 \%$ e $90 \%{ }^{25}$, os achados empíricos evidenciam um contexto adverso ao preconizado:

Eu tive que voltar duas vezes, porque a médica esqueceu de fazer o pedido dos exames. Então, eu fiquei aguardando e, com isso, eu demorei uns 120 dias para fazer os exames. (Participante 8).

Na minha cidade é tudo muito difícil. No bairro com mais ou menos 800 famílias e o médico só passa 2 vezes por semana. Tem um posto que só tem dipirona [...]. (Participante 60).

A médica do posto disse que não tinha nada, porque eu amamentei, e que não poderia ser nada 
[...] Mas tive que insistir foi muito e mostrar o tamanho do caroço para conseguir esse pedido, e ela dizia que era nervosismo meu. No dia seguinte, eu procurei uma clínica com preço popular e fiz meu exame de ultrassonografia de mama. (Participante 62).

Ao analisar os demais serviços utilizados como porta de acesso à rede, verifica-se que o somatório do atendimento na rede privada e suplementar (clínicas, hospitais e emergência) perfaz 47 (33,3\%). Entre os que optaram por serviços de média e alta densidade tecnológica da rede própria e complementar ao SUS (clínicas, policlínicas, unidades de pronto atendimento, maternidades, hospitais públicos), obtiveram-se 26 (18,4\%), seguidos de 15 $(10,6 \%)$ que acessaram as Unacon/Cacon; 10 (7,09\%), a Estratégia Itinerante de Saúde; e 5 (3,54\%), as Campanhas/Mutirões.

Esses achados revelam a multiplicidade de portas de acesso à rede de saúde, com predominância do setor privado não complementar ao SUS. Esse grande número é justificado pela existência de lacunas assistenciais de serviços de apoio diagnóstico, conforme atesta o próprio Plano Estadual de Atenção ao Câncer ${ }^{18}$ e a percepção dos(as) usuários(as) do sentido de urgência que o caso requer, agravada pela demora no processo de regulação do acesso aos serviços, relatada durante as entrevistas.

Eu já era acompanhada no posto de saúde, mas paguei os exames porque não tinha pelo SUS. Para fazer pelo SUS tinha que ir para outra cidade e tem o custo do transporte [...]. (Participante 88).

Procurei atendimento particular, porque o SUS não oferece a vantagem da rapidez. No posto de saúde, eu ia esperar meses. O SUS deixa muito a desejar [...]. (Participante 25).

Fiz meus exames particular, porque é muito difícil pelo SUS e principalmente no interior, que não tem nada a oferecer. Se fosse esperar, eu ia morrer [...]. (Participante 34).
Apesar da constatação, na prática, que a resolutividade da Atenção Básica seja de $80 \%$ a $\mathbf{9 0 \%} \mathbf{2 5}^{\mathbf{2 5}}$, observou-se que as razões para a escolha dos serviços de alta densidade tecnológica como primeiro atendimento, pelos(as) participantes da pesquisa, foram ocasionadas pelo desejo expresso de resolutividade, visto que essas unidades de saúde disponibilizam, em um único local, uma gama variada de serviços, culminando por reforçar a ideia-força do modelo hospitalocêntrico. Sob essa perspectiva, verifica-se a reprodução do modelo médico hegemônico, centrado na doença, no tratamento, no hospital e nos serviços especializados, do que nas ações de prevenção e promoção da saúde, na comunidade, nos territórios e na Atenção Básica ${ }^{23}$. A predominância desse modelo pode acentuar o ciclo vicioso de dependência da ação estatal, favorecendo a lógica de mercado e a financeirização do setor saúde.

Apesar das evidências da eficiência e efetividade dos sistemas públicos universais, quando comparados com os modelos de mercado, a diferença do gasto em saúde entre um setor e outro é expressiva. Enquanto, no Brasil, $54 \%$ do gasto com saúde acontece no setor privado que atende apenas $25 \%$ da população, o SUS conta com apenas $46 \%$ dos recursos para atender $75 \%$ da população ${ }^{26}$. Diante dessa equação, torna-se difícil a APS 'concorrer' em condições de igualdade com os serviços de alta densidade tecnológica.

Os achados empíricos sinalizam uma intencionalidade política de priorização dos serviços de alta densidade tecnológica, concentrados nos prestadores de serviços e/ou organizações sociais de saúde. Ao observar, no Plano Estadual de Saúde (PES) 2016-201927, a previsão alocativa de recursos orçamentários de 62,3\% do Programa Saúde Mais Perto de Você para ampliação dos serviços da Atenção Especializada Ambulatorial e Hospitalar, encontra-se a resposta para a priorização política da atual gestão. 


\section{Sistemas de apoio}

No que se refere aos sistemas de informação de registro do câncer, observa-se alta defasagem, como é o caso do RCBP e RHC, cujos dados datam, respectivamente, dos períodos de 1996-2005 e 1985-201518. Os motivos para tal ocorrência, conforme técnicos que operam os sistemas, são atribuídos às unidades notificadoras, que não lançam os dados de forma regular no sistema, afetando a precisão e a qualidade das informações ${ }^{18}$. Além disso, podem coexistir situações de subnotificação. Por outro lado, os sistemas de informação apontam necessidades de aperfeiçoamento para captura de dados essenciais, sem duplicidade e com interoperabilidade entre eles, conforme observado no estudo empírico.

Concernente ao Siscan, o módulo do Sistema de Informação do Câncer de Mama (Sismama) manteve-se ativo, quando deveria ter sido desabilitado desde a sua implantação, o que tem gerado duas bases de dados sem interoperabilidade, dificultando ainda mais a entrada regular de registros e o levantamento de dados mais fidedignos com a realidade. Ainda sobre o Siscan, verificou-se que não está implantado na maioria dos serviços de mamografia, embora se constitua em um indicador de resultado no eixo diagnóstico no Plano Estadual de Atenção ao Câncer 2016-202318.

A estrutura bipartida da gestão das RAS e dos respectivos sistemas de informação entre áreas distintas, no âmbito da Secretaria da Saúde do Estado da Bahia (Sesab), de acordo com gestores e técnicos, pode comprometer e/ou fragilizar a comunicabilidade entre as equipes, gerar sombreamento ou sobreposições de ações, dificultando, inclusive, o compartilhamento e o acesso integral às informações. Essa foi uma situação vivenciada durante o trabalho de campo.

A defasagem de dados relacionados com o registro de câncer, nos principais sistemas de informação, associada à falta de interoperabilidade, detectada durante o estudo empírico, geram fragmentação e dificultam o gerenciamento e monitoramento dos dados. Além disso, compromete o próprio ato de planejar e definir os desenhos institucionais de políticas públicas mais aderentes ao contexto locorregional.

Embora o Plano Estadual de Atenção ao Câncer ${ }^{18}$ preveja a interconexão entre os pontos de atenção à saúde (módulos ambulatorial e hospitalar), essencial para criar condições propícias para interação da rede interorganizacional, inexiste na realidade pesquisada.

Os dados revelaram o grande nó crítico da gestão da informação no âmbito da saúde pública, enquanto instrumento indispensável para qualificar o processo decisório na formulação/implementação de ações mais efetivas e propiciar a integração sistêmica.

\section{Sistemas logísticos}

Concernente à regulação do acesso aos serviços de saúde, do total de 141 participantes, apenas $26(18,4 \%)$ relataram algum tipo de regulação do sistema municipal para serviços dentro do território de domicílio e/ou para outras unidades de saúde, em âmbito intermunicipal. Referente ao acesso às consultas e/ou exames de diagnóstico, $102(72,3 \%)$ relataram ter utilizado, concomitantemente, a rede assistencial do SUS e a rede privada não complementar; 7 $(4,9 \%)$ utilizaram a rede SUS e suplementar; e $32(22,6 \%)$, somente a rede SUS.

As razões para uso da rede privada não complementar ao SUS, relatadas em subseção anterior, são retomadas neste ponto para caracterizar o processo de regulação de consultas e/ou exames de diagnóstico, no interior e na capital, sobretudo para aquelas(es) que já estavam dentro da rede de atenção secundária e terciária, em alguns casos, em fase preparatória para início de tratamento.

Enfrentei demora na marcação do médico. Não achava vaga, demora nos resultados dos exames e a médica falou para fazer particular para ser mais rápido, mas não tinha meios para fazer e atrasou um pouco meu tratamento [...]. (Participante 1). 
Tive que fazer alguns exames em clínica particular, como RX, porque a máquina aqui estava quebrada, e outros para agilizar o tratamento, pois demora muito [...]. (Participante 26).

\section{[...] tive que fazer o exame de Biópsia em Clínica} Particular, porque precisava ser rápida para a cirurgia [...]. (Participante 37).

Referente ao Tratamento Fora do Domicílio (TFD), do total de 141 participantes, 101 não residiam na capital do estado. Destes, 56 (55,4\%) utilizavam apenas o TFD para acesso às ações e serviços necessários ao tratamento; 24 (23,7\%) combinavam o uso do TFD e recursos próprios para custear os deslocamentos e, assim, acessar a rede de cuidados; 21 (20,79\%) assumiam integralmente os gastos com transporte. Esses dois últimos grupos informaram que usavam recursos próprios, em razão das dificuldades em obter o transporte sanitário. O somatório dos três grupos que relataram algum tipo de dificuldade correspondeu a $62,3 \%$ (63) dos entrevistados.

As dificuldades relatadas pelos dois primeiros grupos para obterem o transporte sanitário foram muito diversificadas, a saber: excesso de demanda, falta de vagas e custos alegados pela Prefeitura; dias limitados para viagens, cujas datas nem sempre coincidiam com o agendamento na unidade de tratamento; irregularidade na garantia do transporte; não reembolso das despesas custeadas pelas(os) usuárias(os); limitação de passagens por mês; não fornecimento de passagem para acompanhantes; o transporte não seguia o roteiro até a unidade de tratamento, obrigando as(os) usuárias(os) a custearem os deslocamentos até o hospital.

Só pelo sangue do cordeiro, porque vim no domingo para ser atendida na quarta e ir embora na sexta-feira. Só tem [transporte] duas vezes por semana. (Participante 27).

Às vezes tem dificuldade e já faltei ao tratamento por causa de carro e tive que usar recursos próprios. (Participante 75).
O valor não é atualizado e a gente tem que complementar com $R \$ 85,00$. Se for com acompanhante, fica $R \$ 170,00$ os dois trechos. É inviável. (Participante 132).

Para a gente que mora no interior, temos o transtorno da viagem. Moro num povoado e, às vezes, não acha carro da Prefeitura. Nunca tive reembolso de passagem. (Participante 136).

O carro deixa as pacientes solta na cidade, e a gente é que tem que pagar o transporte até aqui para o hospital [...]. (Participante 140).

Tem dias que fico no ponto e não me trazem. Às vezes nem passam no ponto. Tem dias que volto de ônibus, porque o carro só vai tarde da noite. Depois da quimioterapia, eu só quero descansar. (Participante 129).

As barreiras sócio-organizacionais impostas às(aos) usuárias(os) para acesso ao TDF ferem o princípio de direito à saúde, reduzindo a obrigatoriedade do poder público municipal a uma ação de cunho facultativo, com atuação seletiva e restritiva, reduzindo as(os) usuárias(os) à condição de subcidadãs(ãos). Tal ação pode comprometer desde a detecção precoce do câncer de mama até o tratamento em tempo oportuno e o seu seguimento, podendo prolongar o período de assistência terapêutica e/ou requerer procedimentos mais invasivos e radicais.

No tocante à variável 'aporte estrutural de acomodações', 41 (50,5\%) participantes informaram fazer uso da casa de apoio disponibilizada pelo município; 22 (21,7\%) e 6 (5,9\%) foram acolhidas(os), respectivamente, em casa de parentes e amigos. Em menor quantitativo, existem aquelas(es) que faziam uso de imóvel alugado ou emprestado; outras(os) utilizaram inicialmente a casa de apoio e posteriormente serviço de hotelaria (pousada ou hotel).

Salienta-se o quantitativo de 27 (26,7\%) usuárias(os) que retornavam diariamente para o município de domicílio. Nesse grupo, estavam aquelas(es) que finalizaram o tratamento de 
radioterapia e encontravam-se em quimioterapia ou estavam em fase de seguimento, com ou sem uso de medicamento específico.

Essa situação desnuda a realidade vivenciada pelas(os) usuárias(os) e revela a face de uma rede interorganizacional com pouca articulação e reduzido grau de interação. As restrições ao acesso comprometem o efetivo cumprimento das competências e responsabilidades sanitárias, além de revelar falhas de ordenamento no fluxo assistencial para atender à demanda, inviabilizando a constituição de ações integradas e tratamento em tempo oportuno.

Cita-se, como limite da pesquisa, o fato de não ter contemplado todos os elementos constitutivos da estrutura operacional das RAS, cujo recorte metodológico ateve-se às variáveis analisadas neste estudo, consideradas representativas da macrogestão, embora não se exclua a possibilidade de realização de outros estudos com os demais componentes da estrutura operacional.

\section{Considerações finais}

O estudo realizado, com o objetivo de analisar a integração sistêmica da atenção à saúde da linha de cuidado do câncer de mama, no contexto da regionalização da saúde no estado da Bahia, sob a perspectiva da macrogestão, mostrou que, embora as normativas do governo federal sejam necessárias, não são suficientes para reduzir as iniquidades do sistema de saúde e as desigualdades regionais, uma vez que a replicação de modelos homogêneos colide com a diversidade e a heterogeneidade da realidade brasileira. Por outro lado, por se tratar de um fenômeno político, as normativas, per si, não são capazes de alterar cenários sociais e políticos institucionais.

Nesse sentido, a morfologia da RAS às pessoas com câncer, no estado da Bahia, sinaliza uma organização de serviços pautada na oferta existente em âmbito locorregional, com vazios assistenciais nos territórios regionais e nos níveis de atenção (média e alta complexidade), cujas lacunas têm sido ocupadas pelo setor privado não complementar ao SUS, em um flagrante processo de transferência da responsabilidade sanitária para as(os) usuárias(os). A configuração do desenho institucional privilegia a contratualização de serviços e a transferência de procedimentos de alto custo para a rede de prestadores, retroalimentando o ciclo de mercantilização do setor saúde e a manutenção do modelo hegemônico médico-centrado privatista.

A incipiente articulação interorganizacional na operacionalização do fluxo de regulação do acesso assistencial identificada na pesquisa contribui para a supremacia do setor privado não complementar ao SUS, haja vista o longo tempo de espera para atendimento, acarretando endividamento financeiro das(os) usuárias(os) e/ou familiares.

Referente à tecnologia de informação e comunicação, a ausência de corredores virtuais, que possibilitem a interconexão e comunicação interorganizacional, a falta de interoperabilidade entre os sistemas de informação, problemas em sua concepção e defasagem dos dados evidenciam que a gestão da informação está longe de ser encarada como relevante ao processo de tomada de decisão, monitoramento e avaliação da rede.

A adoção de estruturas policêntricas para solucionar os complexos problemas da sociedade contemporânea não implica necessariamente relação de cooperação, reciprocidade, comunicação e interdependência, de modo que a integração sistêmica não deve ser tratada como um 'receituário' programático e pragmático, minimizando-se sua natureza política. O processo de negociação e articulação entre os distintos atores é a síntese do propósito de integração sistêmica das RAS, a qual se materializa na ação centrada em âmbito da macro e microgestão do sistema de saúde. Nesse sentido, a integração sistêmica das ações e dos serviços de saúde ainda ocorrem de forma incipiente, com pouca e/ou esparsa articulação entre si. 


\section{Colaboradores}

Landim ELAS (0000-0001-5059-3308)* contribuiu para a concepção, o planejamento, a análise e a interpretação dos dados; revisão crítica do conteúdo; e aprovação da versão final do manuscrito. Guimarães MCL (00000002-1311-8337)* e Pereira APCM (0000-00031940-5254)* contribuíram para revisão crítica do conteúdo e aprovação da versão final.

\section{Referências}

1. Gadelha CAG, Machado CV, Lima LD, et al. Saúde e territorialização na perspectiva do desenvolvimento. Ciênc Saúde Colet. 2011; 16(6):3003-16.

2. Viana ALD, Bousquat A, Pereira APCM, et al. Tipologia das regiões de saúde: condicionantes estruturais para a regionalização no Brasil. Saúde Soc. 2015; 24(2):413-22.

3. Brasil. Ministério da Saúde. Portaria no 4.279, de 30 de dezembro de 2010. Estabelece diretrizes para a organização da Rede de Atenção à Saúde no âmbito do Sistema Único de Saúde (SUS) [internet]. Diário Oficial da União. 31 Dez 2010. [acesso em 2019 nov 6]. Disponível em: http://conselho.saude.gov.br/ultimas_noticias/2011/img/07_jan_portaria4279_301210.pdf.

4. Mendes EV. As redes de atenção à saúde. Ciênc. Saúde Colet. 2010; 15(5):2297-2305.

5. Mendes EV. As redes de atenção à saúde. 2. ed. Brasília: Organização Pan-Americana da Saúde; 2011.

${ }^{*}$ Orcid (Open Researcher and Contributor ID).
6. Viana ALD, Lima LD, Ferreira MP. Condicionantes estruturais da regionalização na saúde: tipologia dos
Colegiados de Gestão Regional. Ciênc. Saúde Colet. 2010; 15(5):2317-26.

7. Queiroz L. A descentralização da gestão: uma abordagem introdutória aos principais desafios postos no âmbito do Sistema Único de Saúde - SUS. In: Tasca $\mathrm{R}$, organizador. Redes e Regionalização em Saúde no Brasil e na Itália: lições aprendidas e contribuições para o debate. Brasília, DF: Organização Pan-Americana da Saúde; 2011. p. 13-25.

8. Brasil. Decreto ${ }^{\circ} 7.508$, de 28 de junho de 2011. Regulamenta a Lei no 8.080, de 19 de setembro de 1990, para dispor sobre a organização do Sistema Único de Saúde - SUS, o planejamento da saúde, a assistência à saúde e a articulação interfederativa, e dá outras providências [internet]. Diário Oficial da União. 29 Jun 2011. [acesso em 2019 nov 6]. Disponível em: http:// www.planalto.gov.br/ccivil_03/_Ato2011-2014/2011/ Decreto/D7508.htm.

9. Ribeiro PT. Perspectiva territorial, regionalização e redes: uma abordagem à política de saúde da República Federativa do Brasil. Saúde Soc. 2015; 24(2):403-12. 
10. Hartz ZMA, Contandriopoulos AP. Integralidade da atenção e integração de serviços de saúde: desafios para avaliar a implantação de um "sistema sem muros”. Cad. Saúde Pública. 2004; 20(supl2):S331-S3.

11. Santos AM, Giovanella L. Gestão do cuidado integral: estudo de caso em região de saúde da Bahia, Brasil. Cad. Saúde Pública. 2016; 32(3):1-15.

12. Pinheiro R, Gerhardt TE, Ruiz ENF, et al. Introdução. O "estado do conhecimento" sobre os itinerários terapêuticos e suas implicações teóricas e metodológicas na Saúde Coletiva e integralidade do cuidado. In: Gerhardt TE, Pinheiro R, Ruiz ENF, et al., organizadores. Itinerários Terapêuticos: integralidade no cuidado, avaliação e formação em saúde. Rio de Janeiro: CEPESC; 2016. p. 13-26.

13. Landim ELAS. Redes de Atenção à Saúde no Contexto da Regionalização: análise da integração sistêmica sob o olhar das(os) usuárias(os) do SUS no estado da Bahia [tese]. Salvador: Universidade Federal da Bahia; 2018. 229 p.

14. Instituto Nacional de Câncer. Estimativa 2018: incidência de câncer no Brasil [internet]. Rio de Janeiro: INCA; 2017 [acesso em 2018 abr 10]. Disponível em: http://pesquisa.bvsalud.org/controlecancer/resources/inc-36228.

15. Bahia. Secretaria da Saúde do Estado da Bahia. Relatório Anual de Gestão da Superintendência de Vigilância e Proteção da Saúde. Salvador: Sesab; 2017.

16. Cunha ABO, Vieira-da-Silva LM. Acessibilidade aos serviços de saúde em um município do Estado da Bahia, Brasil, em gestão plena do sistema. Cad. Saúde Pública. 2010; 26(4):725-37.

17. Brasil. Ministério da Saúde, Conselho Nacional de Saúde. Resolução no 466, de 12 de dezembro de 2012. Aprova as diretrizes e normas regulamentadoras de pesquisas envolvendo seres humanos [internet]. Diário Oficial da União. 13 Jun 2013 [acesso em 2019 nov 8]. Disponível em: https://conselho.saude.gov. br/resolucoes/2012/Reso466.pdf.
18. Bahia. Secretaria da Saúde do Estado da Bahia. Plano Estadual de Atenção ao Câncer 2016-2023. Salvador: Sesab; 2016.

19. Instituto Brasileiro de Geografia e Estatística. População Estimada da Bahia para 2017 [internet]. Brasília, DF: IBGE; 2017 [acesso em 2018 fev 12]. Disponível em: www.ibge.gov.br.

20. Lima LD, Viana ALÁ, Machado CV, et al. Regionalização e acesso à saúde nos estados brasileiros: condicionantes históricos e político-institucionais. Ciênc. Saúde Colet. 2012; 17(11):2881-92.

21. Mendes A, Louvison M. O debate da regionalização em tempos de turbulência no SUS. Saúde Soc. 2015; 24(2):393-402.

22. Viana ALÁ, Bousquat A, Melo GA, et al. Regionalização e Redes de Saúde. Ciênc. Saúde Colet. 2018; 23(6):1791-8.

23. Paim JS. Sistema Único de Saúde (SUS) aos 30 anos. Ciênc. Saúde Coletiva. 2018; 23(6):1723-8.

24. Bahia. Secretaria da Saúde do Estado da Bahia. Cobertura da Estratégia de Saúde da Família [internet]. Salvador: Sesab; 2017 [acesso em 2018 fev 12]. Disponível em: http://www.saude.ba.gov.br/atencao-a-saude/dab/camab/.

25. Santos NR. SUS 30 anos: o início, a caminhada e o rumo. Ciênc. Saúde Coletiva. 2018; 23(6):1729-36.

26. Campos GWS. SUS. O que e como fazer? Ciênc. Saúde Colet. 2018; 23(6):1707-14.

27. Bahia. Secretaria da Saúde do Estado da Bahia. Plano Estadual de Saúde - PES 2016-2019. Salvador: Sesab; 2016.

Recebido em 16/05/2019

Aprovado em 16/10/2019

Conflito de interesses: inexistente

Suporte financeiro: não houve 